\title{
ANÁLISE FÍSICO-QUÍMICA, MICROBIOLÓGICA E SENSORIAL DE FRUTOS DE MAN- GA SUBMETIDOS À DESIDRATAÇÃO OSMÓTICO-SOLAR ${ }^{1}$
}

\author{
MARIA CRISTINA CABRAL BRANDÃO ${ }^{2}$, GERALDO ARRAES MAIA ${ }^{*}$, DORASILVIA PONTES LIMA ${ }^{3}$, EXPEDITO \\ JOSÉ DE SÁ PARENTE ${ }^{3}$, CLAUDIO CABRAL CAMPELLO ${ }^{4}$, RENATA TIEKO NASSU ${ }^{5}$, TEREZINHA FEITOSA ${ }^{4}$, PAU- \\ LO HENRIQUE MACHADO DE SOUSA ${ }^{6}$
}

RESUMO - Polpas de mangas foram submetidas a um processo de secagem solar após pré-tratamento osmótico, com o intuito de obter-se produtos de umidade intermediária. Foram testados quatro pré-tratamentos osmóticos, ou seja, imersão em xaropes de sacarose com $45^{\circ} \mathrm{Brix}, 55^{\circ} \mathrm{Brix}, 65^{\circ} \mathrm{Brix}$ e imersão seqüenciada em xaropes de $45 ; 55$ e $65^{\circ}$ Brix. O processamento envolveu os seguintes passos: desidratação osmótica nas soluções de sacarose adicionadas de substâncias conservantes e secagem solar. Após a secagem, os tratamentos com 45 e $65^{\circ}$ Brix foram selecionados através de análise sensorial para avaliação da estabilidade durante 180 dias de armazenamento à temperatura ambiente $\left(28^{\circ} \mathrm{C}\right)$. Durante todo o processamento e/ ou após a obtenção dos produtos finais, três tipos de avaliações foram realizadas: determinações físico-químicas (atividade de água, pH, acidez total, vitamina $\mathrm{C}$, sólidos solúveis, umidade, açúcar total e açúcares redutores), análises microbiológicas e testes sensoriais. De acordo com os resultados obtidos, os dois produtos apresentaram boa estabilidade no que se refere às propriedades microbiológicas e sensoriais durante o armazenamento. Termos para indexação: Mangifera indica, alimento de umidade intermediária, métodos combinados.

\section{PHYSICAL AND CHEMICAL, MICROBIOLOGICAL AND SENSORIAL ANALYSIS OF MANGO FRUITS SUB- MITTED TO OSMOTIC-SOLAR DEHYDRATION}

\begin{abstract}
Mango pulps were submitted to solar drying processing after osmotic pre-treatment in order to obtain intermediate moisture products. Four osmotic pre-treatments were tested, which were: immersion in sucrose solutions at $45^{\circ}$ Brix, $55^{\circ}$ Brix, $65^{\circ}$ Brix and immersion in sequenced solutions of $45^{\circ}, 55^{\circ}$ and $65^{\circ}$ Brix. The processing involved the following steps: osmotic dehydration in sucrose solutions additioned with conserving substances and solar drying. After drying, the $55^{\circ}$ and $65^{\circ}$ Brix treatments were selected through sensorial analysis for evaluation of the stability during 180 days of storage at room temperature $\left(28^{\circ} \mathrm{C}\right)$. During all the processing and/or after obtaining the final product, it was done three kinds of evaluation, which were: analytic determinations (water activity, $\mathrm{pH}$, total acid, vitamin $\mathrm{C}$, soluble solids, moisture, total sugar, reducing sugars), microbiological analysis and sensorial tests. According to the results obtained, these products have shown good stability regarding to microbiological and sensorial features.
\end{abstract}

Index Terms: Mangifera indica, intermediate moisture food, combined methods.

\section{INTRODUÇÃO}

A manga (Mangifera indica L.) pertence à família Anacardiaceae e figura entre as frutas tropicais de maior expressão econômica nos mercados brasileiro e internacional (Silva et al., 1999). É uma fruta polposa, de aroma e cor muito agradáveis, que faz parte do elenco das frutas tropicais de importância econômica não só pela aparência exótica, mas também por ser uma rica fonte de carotenóides, minerais e carboidratos (Jayaraman, 1988).

A desidratação osmótico-solar vem ganhando interesse, especialmente em países onde existem grande variedade de frutas, ampla disponibilidade de açúcar e alta incidência de raios solares durante todo o ano.

A secagem precedida de tratamento osmótico é uma técnica comumente utilizada na industrialização de alimentos e baseia-se na redução de água disponível para os microrganismos e reações químicas. Foi apontada por vários autores como uma alternativa econômica e segura para a conservação de produtos alimentícios. Esta combinação resulta também em melhores características sensoriais e nutritivas, quando comparadas com os produtos diretamente desidratados. O processo osmótico, como pré-tratamento, consiste na remoção parcial da água pelo uso da pressão osmótica, colocando-se o produto em contato com uma solução concentrada de solutos. Este pré-tratamento promove uma redução no tempo de secagem. A produção de alimentos de umidade intermediária é especialmente indicada para países em desenvolvimento, pois requer tecnologias simples, seus produtos são bastante estáveis sob condições de ambiente, além da economia de energia e baixo capital de investimento
(Fito et al., 1996; Maltini et al., 1991; Torreggiani, 1993).

O presente trabalho teve como objetivo o desenvolvimento de um método de conservação para manga, utilizando-se de secagem solar precedida do processamento osmótico. Foram testadas diferentes concentrações de solutos e avaliaram-se as características físico-químicas tratadas com cada uma destas concentrações. Os alimentos de umidade intermediária produzidos foram analisados durante os 180 dias de armazenagem à temperatura ambiente $\left(28^{\circ} \mathrm{C}\right)$.

\section{MATERIAL E MÉTODOS}

Os frutos de manga da variedade Coité foram adquiridos no mercado varejista de Fortaleza (CE), recepcionados na unidade de processamento e selecionados de acordo com seus atributos de qualidade, como cor, uniformidade, grau de maturação e ausência de injúrias ou doenças. Em seguida, foram lavados com água corrente e permaneceram em água clorada ( 10 ppm de cloro ativo) por 10 minutos.

Estes foram descascados e a polpa cortada manualmente em cubos, que foram branqueados em vapor saturado a $100^{\circ} \mathrm{C}$ por 2 minutos e submetidos ao pré-tratamento osmótico, visando à redução parcial da atividade de água. No tratamento osmótico, foram testadas diferentes concentrações do xarope para imersão dos frutos: $45^{\circ}$ Brix, $55^{\circ}$ Brix, $65^{\circ}$ Brix e tratamento seqüenciado de $45^{\circ}-55^{\circ}-65^{\circ}$ Brix. Nestes xaropes, foram adicionados como conservantes 600 ppm de dióxido de enxofre e 600 ppm de benzoato de sódio, 600 ppm de ácido ascórbico como antioxidante e fonte de vitamina $\mathrm{C}$, e como fonte de íons $\mathrm{Ca}^{+2}$, utilizaram-se $100 \mathrm{ppm}$ de

\footnotetext{
(Trabalho 083/2002). Recebido: 23/04/2002; Aceito para publicação: 21/02/2003. Parte da Dissertação de Mestrado do primeiro autor apresentada a DTA/UFC. Apoio: CAPES.

${ }^{2}$ Eng $^{\circ}$. Alimentos; M. Sc., Universidade Federal do Ceará - UFC.

${ }^{3}$ Professor da Universidade Federal do Ceará - UFC - Rua Silva Jatahy, 400 - ap. 901A - CEP: 60165-070 - Varjota. frutos@ufc.br

${ }^{4}$ Aluno do Doutorado em Bioquímica - Universidade Federal do Ceará - UFC

${ }^{5}$ Pesquisadora da Embrapa Agroindústria Tropical - Rua Dra Sara Mesquita, 2270 - Planalto Pici - Cep: 60511-110 Fortaleza - Ceará - Brasil.

${ }^{6}$ Químico, M. Sc., Dep. Tecnologia de Alimentos, DTA/UFC, phmachado@uol.com.br

* A quem a correspondência deve ser enviada.
} 
cloreto de cálcio; todos os reagentes tinham grau de pureza P.A. Em seguida, os pedaços de cada tratamento foram cozidos a $65^{\circ} \mathrm{C}$ por 15 minutos, seguido de resfriamento até a temperatura ambiente, e manutenção em repouso por 24 horas. $\mathrm{O}$ tratamento seqüenciado sofreu três ciclos de cozimento e repouso (um ciclo após cada imersão em xarope).

Depois do tratamento por osmose, os frutos foram distribuídos em secador solar de construção doméstica dotado de bandejas teladas e sistema de ventilação artificial (ventilador), onde permaneceram por um período variável, entre 48 e 72 horas, até atingir a atividade de água desejada (entre 0,65 e 0,85 ). Os produtos foram retirados e embalados em bandejas de isopor e acondicionados em sacos de polietileno de alta densidade (20 $\mu \mathrm{m}$ de espessura). Em seguida, as embalagens foram termosseladas com retirada parcial de ar e armazenadas à temperatura ambiente $\left(28^{\circ} \mathrm{C}\right)$, sob ventilação e condições higiênico-sanitárias adequadas, para serem submetidas a um estudo de estabilidade por 180 dias. Porém, foi realizado um teste sensorial de aceitação global nos produtos finais, e diante dos resultados sensoriais, optou-se em estudar a estabilidade somente dos tratamentos com $45^{\circ}$ Brix e $55^{\circ}$ Brix, pois os outros dois tratamentos (com $65^{\circ}$ Brix e o seqüenciado) apresentaram rejeição por partes dos provadores.

As determinações realizadas foram: atividade de água (instrumental Aqualab CX-2 Decagon); $\mathrm{pH}$ (pHmetro digital); acidez total titulável expressa em \% de ácido cítrico (Instituto Adolfo Lutz, 1985); teor de dióxido de enxofre total (AOAC 1992); Vitamina C (Pearson, 1976); sólidos solúveis totais em refratômetro de bancada modelo ABEÉ; umidade (AOAC, 1992); açúcares totais pela somatória dos teores de açucares redutores e não-redutores (Adolfo Lutz, 1985); microbiológicas de contagem padrão, bolores e leveduras, coliformes totais e fecais (ICMSF, 1988), e sensorial através de teste de aceitação, utilizando-se de escala hedônica de 9 pontos ancorados em extremos de "gostei muitíssimo" (9) e "desgostei muitíssimo" (1), aplicados a 30 provadores. Os resultados foram avaliados estatisticamente quanto à análise de variância, e a comparação de médias, pelo teste de Tukey. Foi feita utilizando-se o SAS (1996).

\section{RESULTADOS E DISCUSSÃO}

Os valores de $\mathrm{pH}$ não apresentaram variação substancial entre o estado in natura e os produtos processados osmoticamente (Tabela 1); entretanto, houve um aumento na acidez com o uso de solução mais concentrada. Estes resultados diferem do apresentado por Ribeiro \& SabaaSrur (1999), onde se observou semelhante tendência de aumento da acidez, porém associada a uma redução de $\mathrm{pH}$, que foi atribuída a um possível processo de autofermentação. Provavelmente, o uso do ácido cítrico tenha sido a principal causa da elevação no nível de acidez. Os sólidos presentes no xarope podem ter interferido nos resultados de $\mathrm{pH}$, exercendo ação tamponante e evitando a sua alteração.

TABELA 1- Características físicas e químicas de pedaços de polpa de manga analisados em estado in natura e após o pré-tratamento osmótico*.

\begin{tabular}{lccccc}
\hline Determinações analíticas & in natura & $\mathrm{T}_{\mathrm{I}}$ & $\mathrm{T}_{\mathrm{II}}$ & $\mathrm{T}_{\mathrm{III}}$ & $\mathrm{T}_{\mathrm{IV}}$ \\
\hline $\mathrm{pH}$ & 3,60 & 3,65 & 3,68 & 3,68 & 3,83 \\
Acidez total titulável & 0,61 & 0,75 & 0,77 & 0,89 & 0,95 \\
(\% ácido cítrico) & & & & & \\
Teor de $\mathrm{SO}_{2}(\mathrm{ppm})$ & - & 232,62 & 225,00 & 235,00 & 448,52 \\
Vitamina C (mg/100g) & 18,00 & 61,00 & 59,31 & 64,13 & 81,78 \\
Sólidos solúveis ( ${ }^{\circ}$ Brix) & 16,00 & 33,70 & 35,00 & 37,20 & 60,00 \\
Umidade (\%) & 83,03 & 69,57 & 62,52 & 59,12 & 32,64 \\
Açúcar total (\%) & 12,20 & 28,99 & 30,00 & 29,58 & 38,85 \\
Açúcares redutores & 3,80 & 3,28 & 3,70 & 4,06 & 3,07 \\
(\% glicose) & & & & & \\
Atividade de água & 0,982 & 0,961 & 0,955 & 0,944 & 0,874 \\
\hline
\end{tabular}

* Tratamentos I (xarope de $45^{\circ}$ Brix), II (xarope de $55^{\circ}$ Brix) e III (xarope de 65 ${ }^{\circ}$ Brix), IV (imersões sucessivas em 45 ; 55 e $65^{\circ}$ Brix).
Quanto à retenção de $\mathrm{SO}_{2}$ pelos pedaços de polpa de manga pré-tratados, foi verificada uma tendência de reter-se aproximadamente $40 \%$ nos tratamentos simples e $75 \%$ no seqüenciado (Tabela 1 ). Valores mais altos no tratamento seqüenciado eram esperados, em decorrência da quantidade residual retida após cada imersão em xarope (Fennema, 1993; Mesquita, 1999; Pina, 1999).

A manga em pedaços apresentava teores de vitamina $\mathrm{C}$ de $18,0 \mathrm{mg} .100 \mathrm{~g}^{-1}$, que aumentou para $59-64 \mathrm{mg} \cdot 100 \mathrm{~g}^{-1}$ com os tratamentos, com exceção do tratamento seqüencial em xaropes, no qual a imersão produziu um somatório final de $82 \mathrm{mg} \cdot 100 \mathrm{~g}^{-1}$, que se deve à concentração desta vitamina devido à maior retirada de água neste tratamento (Tabela 1).

Os teores de sólidos solúveis, que na manga eram de $16,0^{\circ}$ Brix, aumentaram com o tratamento em xaropes a $45^{\circ}$ Brix, $55^{\circ}$ Brix e $65^{\circ}$ Brix para 33-37 Brix, observando-se discreta elevação com o aumento da concentração do xarope. $\mathrm{O}$ teor nos pedaços após o tratamento seqüenciado foi quase o dobro $\left(60^{\circ}\right.$ Brix $)$ do valor nos frutos tratados com imersões simples (Tabela 1). Este resultado era esperado, pois a imersão sucessiva em xaropes concentrados de sacarose acarreta maior ganho de sólidos e maior perda de água (Mesquita, 1999; Pina, 1999).

O teor de umidade dos pedaços, que era de $83,03 \%$, reduziu-se para $59-69 \%$ com os produtos tratados com os xaropes simples, enquanto a atividade de água se reduziu de 0,982 para $0,944-0,961$. Tais resultados encontram-se dentro da faixa esperada para alimentos de alta umidade (Chirife \& Faveto, 1992). Entretanto, a umidade verificada após o tratamento seqüenciado foi bem menor $(32,6 \%)$, assim como a atividade de água $(0,874)$, devido ao aumento do tempo de desidratação e à exposição dos frutos à seqüência de xaropes concentrados (Tabela 1).

Não houve alteração acentuada no teor de açúcares redutores com o pré-tratamento osmótico, o que pode ser creditado às características do peso específico dos açúcares redutores e à permeabilidade das membranas celulares à penetração destes açúcares (Ribeiro \& SabaaSrur, 1999). Porém, os valores correspondentes ao teor de açúcar total aumentaram de $12,2 \%$ para $28-30 \%$ nos tratamentos com imersões simples, e para $39 \%$ no tratamento seqüenciado, no qual houve aumento gradativo na concentração de sacarose (Tabela 1). Trabalhos realizados anteriormente demonstraram haver aumento no conteúdo de açúcares redutores em função do tratamento térmico e do aumento da acidez, que contribuem para que ocorra a inversão da sacarose durante a osmose (Mesquita, 1999; Pina, 1999).

Logo após a secagem, foi realizada a análise microbiológica dos produtos, a qual revelou baixos valores de contagem padrão $(<10 \mathrm{UFC} / \mathrm{g})$, bolores e leveduras $(<10 \mathrm{UFC} / \mathrm{g})$ e coliformes totais e fecais $(<3 \mathrm{NMP} / \mathrm{g})$. Tais resultados estão de acordo com a legislação vigente (Brasil, 2001).

Os produtos com umidade intermediária foram submetidos à análise sensorial, com o intuito de avaliar-se o grau de aceitação global pelos potenciais consumidores. A análise sensorial (aceitação global) não mostrou diferença significativa $(\mathrm{p} \leq 0,05)$ entre os produtos (Tabela 2). O produto proveniente do tratamento IV, ou imersão seqüenciada, não foi incluído no painel em virtude de problemas com a sua textura, excessivamente rígida. Os provadores demonstraram ainda, em caráter voluntário, insatisfação com o sabor doce acentuado e a textura rígida dos produtos com o tratamento a $65^{\circ}$ Brix (Tratamento III). Com base nesses resultados, foram eleitas somente as duas amostras mais atrativas (TI e TII) para a avaliação da estabilidade por 180 dias.

TABELA 2- Médias da avaliação sensorial (aceitação global) de pedaços de polpa de manga pré-tratados osmoticamente* e submetidos à secagem.

\begin{tabular}{lcccc}
\hline Material & $\mathrm{T}_{\mathrm{I}}$ & $\mathrm{T}_{\mathrm{II}}$ & $\mathrm{T}_{\mathrm{III}}$ & $\mathrm{T}_{\mathrm{IV}}$ \\
\hline Manga & $7,2^{\mathrm{a}}$ & $7,5^{\mathrm{a}}$ & $7,5^{\mathrm{a}}$ & $\mathrm{nd}^{* *}$ \\
\hline
\end{tabular}

$* \mathrm{~T}_{\mathrm{I}}=$ xarope de $45^{\circ}$ Brix, $\mathrm{T}_{\mathrm{II}}=$ xarope de $55^{\circ} \mathrm{Brix}, \mathrm{T}_{\mathrm{III}}=$ xarope de $65^{\circ} \mathrm{Brix}, \mathrm{T}_{\mathrm{IV}}=$ seqüenciado; $* *$ nd $=$ não determinado.

Médias dentro da mesma linha, com a mesma letra, não são significativamente diferentes, no nível de 5\%, pelo teste de Tukey. 
Os valores de pH não apresentaram variação substancial entre os dois produtos após a secagem. Os valores relativos à acidez total titulável também foram semelhantes para os dois produtos (Tabela 3 ).

TABELA 3- Características físicas e químicas de pedaços de polpa de manga analisados após a secagem solar e após 180 dias de estocagem.

\begin{tabular}{lcccc}
\hline \multirow{2}{*}{ Determinações analíticas } & \multicolumn{4}{c}{ Tratamentos* } \\
\cline { 2 - 5 } & \multicolumn{3}{c}{ TI } & \multicolumn{3}{c}{ TII } \\
\cline { 2 - 5 } & 0 & 180 & 0 & 180 \\
\hline PH & 3,50 & 3,40 & 3,40 & 3,50 \\
Acidez total titulável (\% ácido cítrico) & 1,40 & 1,35 & 1,40 & 1,50 \\
Teor de $\mathrm{SO}_{2}(\mathrm{ppm})$ & 263,25 & 0,00 & 185,36 & 0,00 \\
Vitamina C (mg/100g) & 82,960 & 0,000 & 96,830 & 0,000 \\
Sólidos solúveis ( ${ }^{\circ}$ Brix) & 66,860 & 70,530 & 74,000 & 71,730 \\
Umidade (\%) & 27,400 & 23,370 & 21,400 & 19,400 \\
Açúcar total (\%) & 65,160 & 60,230 & 63,620 & 57,800 \\
Accúcares redutores (\% glicose) & 16,260 & 32,970 & 15,370 & 26,300 \\
Atividade de água & 0,820 & 0,740 & 0,753 & 0,710 \\
\hline
\end{tabular}

* TI (xarope de $45^{\circ}$ Brix) e TII (xarope de $55^{\circ}$ Brix).

A retenção de $\mathrm{SO}_{2}$ nos pedaços de manga diferiu consideravelmente entre os produtos secos após imersão em xarope com $45^{\circ}$ Brix e $55^{\circ}$ Brix (Tabela 3). Comparados com os dados obtidos logo após o prétratamento osmótico, verifica-se que os valores são bastante similares. Já, após 180 dias de armazenagem, os valores de $\mathrm{SO}_{2}$ chegaram a zero nos dois tratamentos, o que se deve à degradação química do mesmo.

$\mathrm{O}$ teor de vitamina $\mathrm{C}$ aumentou no produto final desidratado; com umidade intermediária, foi comparando aos dados obtidos do fruto em estado in natura (Tabela 3). A diferença entre os teores de vitamina $\mathrm{C}$ nos dois tratamentos analisados foi de pequena magnitude. Após 180 dias de armazenamento, o valor de vitamina $\mathrm{C}$ chegou a zero, o mesmo acontecendo com Alzamora et al. (1989) em estudo com abacaxi processado por métodos combinados, em que o teor inicial de $15,35 \mathrm{mg} / 100 \mathrm{~g}$ de ácido ascórbico, após 180 dias de armazenamento, chegou a zero, sendo devido à degradação química da mesma.

O aumento nos teores de sólidos solúveis durante o armazenamento pode ser atribuído ao efeito da incorporação de sólidos e à concentração do xarope. Os resultados referentes à umidade e atividade de água após a secagem solar encontram-se dentro dos padrões estabelecidos para produtos de umidade intermediária (Tabela 3) (Chirife \& Faveto, 1992; Karel, 1975).

Os teores de açúcares totais e açúcares redutores aumentaram nos frutos de manga submetidos à secagem após o pré-tratamento osmótico (Tabelas 2 e 3). Este fato pode ser atribuído ao efeito da remoção de água em função da secagem.

Uma nova análise sensorial foi realizada somente nos tratamentos selecionados para estudo da estabilidade (com $45^{\circ}$ Brix e $55^{\circ}$ Brix) para caracterizar de forma mais precisa o material recém-processado com o objetivo de obter-se dados para avaliar o produto sob condições de armazenamento.

Os pedaços de manga processados obtiveram média de aceitação entre 6 e 7 na escala hedônica, que correspondem a "gostei ligeiramente" e "gostei moderadamente". A análise feita pelo teste de Tukey revelou não ter havido diferença significativa $(p \leq 0,05)$ entre as amostras (Tabela 4).

A textura resultante de mudanças ocorridas nas substâncias pécticas da parede celular do tecido vegetal, associada à doçura dada pela relação Brix/acidez, conferiu características agradáveis ao produto final. A aparência foi provavelmente favorecida pelo brilho característico dos produtos açucarados de umidade intermediária, superior aos produtos completamente desidratados. Estes resultados encontram concordância com estudos realizados anteriormente por Aska et al. (1996), que avaliaram a ação da osmose e da secagem solar sobre pedaços de frutas e sobre o seu efeito positivo na cor e sabor dos produtos desidratados. A osmose reduz o conteúdo de água e promove a melhoria das características textura, aroma e aumento no teor de açúcar da fruta.

TABELA 4- Médias das notas atribuídas pelos provadores no teste de aceitabilidade de pedaços de polpa de manga submetidos ao processamento osmótico / solar, com $45^{\circ}$ Brix (T1) e $55^{\circ}$ Brix (T2), durante 180 dias de armazenagem à temperatura ambiente $\left(28^{\circ} \mathrm{C}\right)$.

\begin{tabular}{lcccccccccc}
\hline Tempo & \multicolumn{3}{c}{$\mathrm{T}=0^{*}$} & \multicolumn{2}{c}{$\mathrm{T}=45$} & \multicolumn{2}{c}{$\mathrm{T}=90$} & \multicolumn{2}{c}{$\mathrm{T}=135$} & \multicolumn{2}{c}{$\mathrm{T}=180$} \\
\hline Amostras & TI & TII & TI & TII & TI & TII & TI & TII & TI & TII \\
\hline Aparência & $7,0^{\mathrm{a}}$ & $6,6^{\mathrm{a}}$ & $5,9^{\mathrm{a}}$ & $6,4^{\mathrm{a}}$ & $4,6^{\mathrm{b}}$ & $6,1^{\mathrm{a}}$ & $5,0^{\mathrm{a}}$ & $5,2^{\mathrm{a}}$ & $6,2^{\mathrm{a}}$ & $6,0^{\mathrm{a}}$ \\
Aroma & $6,6^{\mathrm{a}}$ & $6,4^{\mathrm{a}}$ & $6,4^{\mathrm{a}}$ & $6,1^{\mathrm{a}}$ & $5,9^{\mathrm{a}}$ & $6,6^{\mathrm{a}}$ & $5,8^{\mathrm{a}}$ & $5,7^{\mathrm{a}}$ & $6,2^{\mathrm{a}}$ & $6,4^{\mathrm{a}}$ \\
Sabor & $7,4^{\mathrm{a}}$ & $7,4^{\mathrm{a}}$ & $7,0^{\mathrm{a}}$ & $7,1^{\mathrm{a}}$ & $6,4^{\mathrm{a}}$ & $7,2^{\mathrm{a}}$ & $6,6^{\mathrm{a}}$ & $9,2^{\mathrm{a}}$ & $7,5^{\mathrm{a}}$ & $7,1^{\mathrm{a}}$ \\
Textura & $6,7^{\mathrm{a}}$ & $6,6^{\mathrm{a}}$ & $6,6^{\mathrm{a}}$ & $6,3^{\mathrm{a}}$ & $6,2^{\mathrm{a}}$ & $6,2^{\mathrm{a}}$ & $6,1^{\mathrm{a}}$ & $6,3^{\mathrm{a}}$ & $7,2^{\mathrm{a}}$ & $6,9^{\mathrm{a}}$ \\
Aceitação & $6,9^{\mathrm{a}}$ & $7,0^{\mathrm{a}}$ & $6,6^{\mathrm{a}}$ & $6,7^{\mathrm{a}}$ & $6,0^{\mathrm{a}}$ & $6,9^{\mathrm{a}}$ & $6,3^{\mathrm{a}}$ & $6,5^{\mathrm{a}}$ & $6,9^{\mathrm{a}}$ & $6,84^{\mathrm{a}}$
\end{tabular}

Global

*T $=0$ amostra recém-processada; $\mathrm{TI}=$ tratamentos a $45^{\circ} \mathrm{Brix} ; \mathrm{TII}=$ tratamento a $55^{\circ}$ Brix.

Médias dentro da mesma linha, com a mesma letra, não são significativamente diferentes, no nível de 5\%, pelo teste de Tukey.

Durante a estocagem, os resultados microbiológicos apresentaram valores para contagem padrão, $<10 \mathrm{UFC/g}$, bolores e leveduras, $<$ $10 \mathrm{UFC} / \mathrm{g}$ e coliformes totais e fecais, $<3 \mathrm{NMP} / \mathrm{g}$. Estão, portanto, dentro dos padrões estabelecidos pela legislação vigente (Brasil, 2001). Tais resultados demonstram que a armazenagem de 180 dias à temperatura ambiente $\left(28^{\circ} \mathrm{C}\right)$ não afetou o número de microrganismos e confirmam dados de estudos anteriores (Jamayaraman, 1988).

Os atributos aroma, sabor, textura e aceitação global também não apresentaram diferenças significativas $(\mathrm{p} \leq 0,05)$ para os dois produtos de manga em função do tempo de estocagem (Tabela 4). Flutuações entre as notas durante o período de estocagem podem ser explicadas pelo fato de a equipe de provadores não ter sido treinada para análise destes produtos especificamente.

Entre os produtos desidratados de manga, não houve diferença significativa $(\mathrm{p} \leq 0,05)$ em relação aos atributos aroma, sabor, textura e aceitação global (Tabela 4).

Estes resultados demonstram a validade do processo osmóticosolar e estabilidade durante a armazenagem, confirmando a efetividade da redução da atividade de água combinada com o uso de conservantes e com a redução do $\mathrm{pH}$ para inibição da proliferação de microrganismos. Estes resultados confirmam observações feitas por Jayaraman (1988), que considerou os produtos de umidade intermediária como estáveis à temperatura ambiente, podendo ser conservados sem necessidade de refrigeração.

\section{CONCLUSÕES}

Os resultados obtidos neste trabalho permitem concluir que os processo selecionados (TI e TII) para compor o estudo da estabilidade dos produtos foram efetivos na conservação dos produtos, considerando-se que, em todas as etapas dos experimentos, os resultados microbiológicos foram satisfatórios a ponto de serem indicados como método de conservação de manga. Desse modo, os produtos finais poderiam ser incluídos na categoria de alimentos de umidade intermediária, passíveis de utilização como ingredientes para sobremesas e gelados comestíveis.

\section{REFERÊNCIASBIBLIOGRÁFICAS}

ALZAMORA, S. M.; GERSCHENSON, L. N.; CERRUTI, P.; ROJAS, A. $M$. Shelf stable pineapple for long term non-refrigerated storage. Lebensmittel Wissenschaft \& Technologie, London, v. 22, p. 233236, 1989.

AOAC - Association of Official Analytical Chemists - Official Methods

of Analysis of the AOAC. 10.ed. Washington, 1992. $1115 \mathrm{p}$. ASKA, A; HEIKAI, Y; GHONAAIN, S. M.; ABDEL-FADEELM.G.;ALI, 
A M.; ABD EL-GAIED, I.O. Osmotic and solar dehydration of peach fruits. Fruit Processament, v.7, p.259-261, 1996.

BRASIL. Resolução RDC - nº 12 de 02 de janeiro de 2001. Dispõe sobre os princípios gerais para o estabelecimento de critérios e padrões microbiológicos para alimentos. Brasília: Agência Nacional de Vigilância Sanitária-ANVISA, 2001.

CHIRIFE, J.; FAVETO, G.J. Some physico-chemical basis of food preservation by combined methods. Food Research International, Kidlington, v.25, n.5, p.389-396, 1992.

FENNEMA, O. R. Química de los alimentos. 2 ed. Zaragoza, España: Acribia, 1993. 1094p.

FITO, P. et al. Coupling of hydrodynamic mechanism and deformationrelaxation phenomena during vacuum treatments in solid porous foodliquid systems. Journal of Food Engineering, Kidlington, v. 21, p. 229-240, 1996.

ICMSF (International Commission on Microbiological Specifications for Foods). Microorganisms in foods 1 - Their significance and methods of enumeration. 2.ed. Toronto: University of Toronto Press, 1988, 436p.

INSTITUTO ADOLFO LUTZ. Métodos químicos e físicos para análise de alimentos. 3.ed. São Paulo: Normas analíticas do Instituto Adolfo Lutz, 1985. v. 1,553p.

JAYARAMAN, K.S. Development of intermediate moisture tropical fruits and vegetable products. Technological problems and prospects. SEOW, C.C. Applied Sciences. Essex: Elsevier, 1988. 175p.

KAREL, M. Dehydration of food. In: Principles of food science. Part II.
Physical principles of food preservation. Marcel Dekker, Inc, New York, 1975.

MALTINI, E.; TORREGGIANI, D.; FORNI, E. et al. Osmotic properties of fruit juice concentrates. In: SPIESS, W.L.E.; SCHUBERT, H. Engineering and food, physical properties and process control; London: Elsevier Science Publishing Company, 1990, v. 1, p. 567-573.

MESQUITA, P.C. Conservação do pedúnculo de caju (Anacardium occidentale $\mathrm{L}$.) por processamento mínimo/métodos combinado com emprego de tecnologia de obstáculos. 1999, 125f. Dissertação (Mestrado em Tecnologia de Alimentos) - Universidade Federal do Ceará, Fortaleza, 1999.

PEARSON, D. Técnicas de laboratório para el análises de alimentos. Zaragoza, España: Acribia, 1976.331p.

PINA, M.M.G. Conservação de manga (Mangifera indica, L.) por processamento mínimo/métodos combinado. 1999, 138f. Dissertação (Mestrado em Tecnologia de Alimentos) - Universidade Federal do Ceará, Fortaleza, 1999.

RIBEIRO, M.S.; SABAA-SRUR, A. U. O. Saturação de Manga (Mangifera indica L.), var. rosa com açúcares. Ciência e Tecnologia de Alimentos, Campinas, v.19, n.1, 1999.

SAS Institute, Inc. SAS user's Guide: Statistical Analysis System, Cary, N.C., 1996.

SILVA, E.M.F. (Coord.); AMARAL, C.M.; CARMO, H.C.E.; MAURY, P.M. Estudos sobre mercado de frutas. São Paulo: FIPE, 1999, 373p.

TORREGGIANI, D. Osmotic dehydration in fruit and vegetable processing. Food Research International, Kidlington, v. 26, p. 59-68, 1993. 